\title{
Selection of Pseudomonas putida Strains with Elevated Dehalogenase Activities by Continuous Culture Growth on Chlorinated Alkanoic Acids
}

\author{
By ANDREW J. WEIGHTMAN AND J. HOWARD SLATER* \\ Department of Environmental Sciences, University of Warwick, Coventry CV4 $7 A L$
}

(Received 30 April 1980)

\begin{abstract}
A continuous-flow culture selection experiment was designed to obtain mutants of Pseudomonas putida strain PP3 capable of growing on 2-monochlorobutanoic acid (2MCBA), normally unable to act as a carbon and energy source for $P$. putida PP3. The experiment involved continuous exposure of $P$. putida PP3 to 2MCBA whilst the population's growth was supported by the metabolizable substrate, 2-monochloropropionic acid. The system failed to select for mutants able to grow on 2MCBA alone. However, the procedure resulted in the selection of two different strains of P. putida, namely, PP309 and PP310, both stably maintained as a mixed culture having excluded the parent strain. The two mutants differed significantly from $P$. putida PP3 with respect to their dehalogenating system since it was found that major changes in both dehalogenase specific activities and overall activity ratios had occurred. The dehalogenase system in P. putida strains PP309 and PP310 was inducible as in the parent strain. The results obtained were explained in terms of elevated production of fraction I dehalogenase compared with fraction II dehalogenase in the mutant strains in contrast to the parent strain. The results demonstrate that mutants may be selected showing altered enzyme activities in the absence of acquiring the capacity to grow solely on a novel substrate which provided the selection pressure.
\end{abstract}

\section{INTRODUCTION}

Pseudomonas putida PP3 is able to grow on some halogenated alkanoic acids by virtue of its dehalogenating capability (Slater et al., 1979). It has recently been demonstrated that this strain contains at least two different dehalogenases (Weightman et al., 1979a, $b$ ). The fraction I dehalogenase has most activity against 2-monochloropropionic acid and 2,2dichloropropionic acid, whilst fraction II dehalogenase utilizes dichloroacetic acid as its principal substrate. Both enzymes have an approximately equal activity against monochloroacetic acid. Furthermore, studies with the partially purified dehalogenases have shown that other halogenated alkanoic acids can serve as substrates for these enzymes, albeit at significantly reduced rates of dehalogenation compared with the four major substrates. Thus, it has been suggested that these enzymes could be useful experimental systems for examining the acquisition of novel catalytic functions coupled with the capacity to grow on new compounds (Bull et al., 1976). A number of different categories of substrates can be considered which may be important so far as enzyme-substrate specificity of the existing dehalogenases is concerned. Firstly, 2-substituted alkanoic acids with increasing carbon chain length might be less successful substrates for the existing enzymes. Indeed, a sufficiently long carbon chain length might result in no activity with the existing dehalogenases. Secondly, the nature of the halogen on the 2-carbon position might be varied with respect to both its halogen species and its stereochemical configuration, producing substrates which 
may or may not be dechlorinated. Thirdly, the position of the halogen substitution on the alkanoic acid could be altered producing another series of potential substrates. Previous studies have indicated that fraction I and II dehalogenases have little or no activity for halogenated alkanoic acids other than those substituted in the 2-carbon position (Slater et al., 1979). The nature of the halogen at the 2-carbon position, certainly so far as brominated compounds are concerned, has little effect on dehalogenase activity (A. J. Weightman \& J. H. Slater, unpublished observations). However, variation in the carbon chain length of the alkanoic acids produces an intermediate response so that, for example, 2-monochlorobutanoic acid (2MCBA) is dechlorinated by $P$. putida PP3 at approximately $45 \%$ of the 2-monochloropropionic acid dehalogenation rate (Weightman et al., 1979b). We have demonstrated that greater than $80 \%$ of the 2MCBA dehalogenating activity was associated with the fraction I dehalogenase. Pseudomonas putida PP3 does not utilize 2MCBA as a growth substrate, possibly because the 2MCBA dehalogenase activity is inadequate to support growth, although the compound does induce both fraction I and II dehalogenases (Weightman et al., 1979b).

This study reports experiments designed to select strains of Pseudomonas putida capable of growing on 2MCBA using continuous-flow culture selection techniques with attention focused on alterations in the dehalogenase activities.

\section{METHODS}

Abbreviations. MCA, Monochloroacetic acid; DCA, dichloroacetic acid; 2MCPA, 2-monochloropropionic acid; 22DCPA, 2,2-dichloropropionic acid; 2MCBA, 2-monochlorobutanoic acid.

Sources and cultivation of Pseudomonas putida strains. Pseudomonas putida PP3 (previously designated P3) was isolated from a stable microbial community enriched for by continuous-flow culture techniques with the herbicide Dalapon (22DCPA) as the limiting carbon and energy source (Senior et al., 1976). The culture maintenance and the basic growth media were the same as previously described (Slater et al., 1979).

The continuous-flow selection experiments were undertaken using LH Engineering Series 500 Modular Fermenter Units (LH Engineering, Stoke Poges, Bucks.). The culture volume was $800 \mathrm{ml}$, agitated at $1000 \mathrm{rev} . \mathrm{min}^{-1}$ and aerated at the rate of $800 \mathrm{ml}$ air $\mathrm{min}^{-1}$. The culture was maintained at $30 \pm 0.5^{\circ} \mathrm{C}$ and fresh medium was pumped into the vessel using flow inducers (Watson-Marlow, Falmouth, Cornwall). A dilution rate of $D=0.075 \mathrm{~h}^{-1}$ was used throughout the selection experiment. The basic growth medium was supplemented with a mixture of filter-sterilized 2MCPA to a final concentration of $0.20 \mathrm{~g} \mathrm{C}^{-1}(0.60 \mathrm{~g}$ 2MCPA $\left.1^{-1} ; 5.56 \mathrm{mM}\right)$ and filter-sterilized 2MCBA to a final concentration of $0.50 \mathrm{~g} \mathrm{C}^{-1}(1.28 \mathrm{~g} \mathrm{2MCBA}$ $1^{-1} ; 10.42 \mathrm{~mm}$ ). Assuming complete utilization of both these compounds the combined carbon source represented the growth-limiting substrate. Periodically, the culture absorbance was determined at $600 \mathrm{~nm}$ using a Pye Unicam SP1700 spectrophotometer.

Strain purity. The purity of the culture and isolated strains was regularly checked by plating out on nutrient agar, minimal agar containing 2MCPA, and King's B agar, the last medium being used as a diagnostic check for $P$. putida (King \& Phillips, 1978).

Chloride ion determination. Substrate dechlorination in both culture media and dehalogenase assays was monitored by measuring free chloride ion concentrations using a Marius Chlor-o-Counter (Labo International, Holland) as described previously (Slater et al., 1979).

Measurement of dehalogenase activity. At appropriate times the dehalogenase activity in the chemostat culture was determined. A 600 to $700 \mathrm{ml}$ sample of culture was harvested and cell-free extract was prepared as described previously (Slater et al., 1979). Assay tubes contained a total volume of $5.5 \mathrm{ml}$ with $900 \mu \mathrm{mol}$ Tris/sulphate buffer pH 7.9, $165 \mu \mathrm{mol}$ chlorinated substrate (MCA, DCA, 2MCPA, 22DCPA or 2MCBA) and 0.10 to $0.75 \mathrm{ml}$ cell-free extract. The assay mixtures were incubated at $30^{\circ} \mathrm{C}$ and $1 \mathrm{ml}$ samples were assayed for free chloride ions at appropriate intervals. A unit of activity is defined as that amount of enzyme which converts $1 \mu \mathrm{mol}$ substrate to product $\mathrm{min}^{-1}$. The products are glycollate, glyoxylate, lactate, pyruvate, and 2-hydroxybutanoic acid for MCA, DCA, 2MCPA, 22DCPA and 2MCBA, respectively. Protein was determined using the Biuret method (Gornall et al., 1948).

Electrophoresis. An analytical disc polyacrylamide gel electrophoresis technique, based on those of Laemmli (1970) and Fairbanks $e$ t al. (1971), was used to separate the dehalogenases in cell-free extracts. The running gels contained $0.325 \mathrm{M}$-Tris/sulphate buffer $\mathrm{pH} 8 \cdot 8,1 \mathrm{~mm}$-dithiothreitol, $80.0 \mathrm{~g}$ acrylamide $\mathbf{1}^{-1}$,

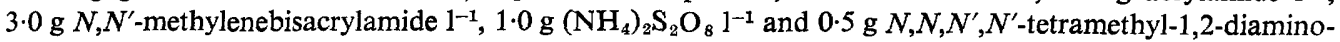
ethane (TEMED) $1^{-1}$. The stacking gels contained $0.125 \mathrm{M}$-Tris/sulphate buffer $\mathrm{pH} 6 \cdot 8,30 \cdot 0 \mathrm{~g}$ acrylamide $1^{-1}$, 


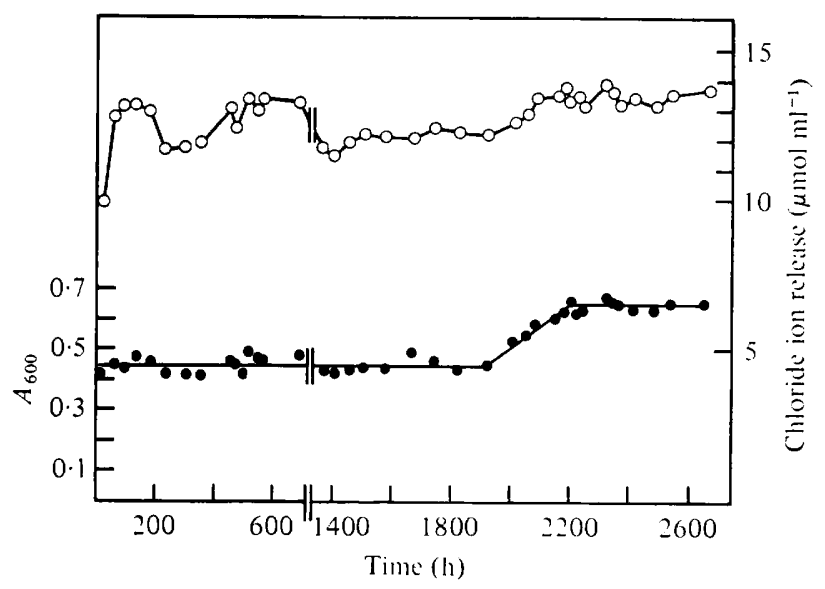

Fig. 1. Continuous-flow selections of Pseudomonas putida, at $D=0.075 \mathrm{~h}^{-1}$. Media contained 2MCPA at $0.20 \mathrm{~g} \mathrm{C}^{-1}$ and $2 \mathrm{MCBA}$ at $0.50 \mathrm{~g} \mathrm{C}^{-1}$. Chloride ion release (O). Culture $A_{600}(O)$.

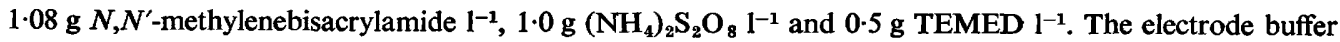
contained $0.025 \mathrm{M}$-Tris, $0.192 \mathrm{M}$-glycine and $1 \mathrm{mM}$-dithiothreitol. The slab gels were run at $25 \mathrm{~mA}$ for about $5 \mathrm{~h}$ at $4^{\circ} \mathrm{C}$.

Dehalogenase bands were visualized using the following staining technique. The gels were incubated in $0.2 \mathrm{M}$-Tris/sulphate buffer $\mathrm{pH} 7.9$, at $30^{\circ} \mathrm{C}$ in the presence of $50 \mathrm{~mm}$ halogenated substrate for approximately $20 \mathrm{~min}$ and then washed in water. Chloride ions released during this process were precipitated by the addition of $0 \cdot 1 \mathrm{M}-\mathrm{AgNO}_{3}$, and thus the positions of dehalogenases in the gels were indicated by the precipitated $\mathrm{AgCl}$ bands.

\section{RESULTS}

The continuous-flow culture selection experiment was established on the principle that the Pseudomonas putida PP3 population could be maintained in the culture system as a result of growth on 2MCPA and, at the same time, be continuously exposed to a high concentration of a compound, 2MCBA, which did not support growth. Under these conditions a suitable selection pressure was applied which, it was anticipated, would favour the evolution of a mutant population able to use 2MCBA.

For the first $1900 \mathrm{~h}$ of growth, the culture absorbance was constant at a level close to that expected for a population growing only on 2MCPA (Fig. 1). However, the chloride ion concentration showed some variation with an average value between 13 and $14 \mu \mathrm{mol} \mathrm{Cl}-$ $\mathrm{ml}^{-1}$ indicating that both 2MCPA and 2MCBA were dehalogenated. A maximum chloride ion concentration of $15.98 \mu \mathrm{mol} \mathrm{Cl}-\mathrm{ml}^{-1}$ would have been expected if both halogenated alkanoic acids were fully dehalogenated, but a maximum of only $5.56 \mu \mathrm{mol} \mathrm{Cl}^{-} \mathrm{ml}^{-1}$ if only 2MCPA was completely dehalogenated. These results showed that approximately $85 \%$ of the combined substrates were dehalogenated and further suggested that the product of $2 \mathrm{MCBA}$ dechlorination, probably 2 -hydroxybutanoic acid, was not utilized as a carbon source. A marked increase in organism biomass, observed as a $45 \%$ increase in culture absorbance, was recorded between 1900 and $2200 \mathrm{~h}$ of continuous growth without a significant change in chloride ion concentration (Fig. 1). This period was characterized by changes in organism colony morphology leading to the recognition of two types which were stably maintained together in the culture vessel. One strain, P. putida PP310, was very similar to strain PP3, producing characteristic gelatinous colonies on King's B medium. The second, P . putida PP309, produced smaller, less gelatinous colonies on both King's B and 2MCPA media.

After $2500 \mathrm{~h}$ of growth, the dehalogenase activities in cell-free extracts from the chemostat 
Table 1. Dehalogenase specific activities in Pseudomonas putida strains from continuous-flow and closed culture

\begin{tabular}{|c|c|c|c|c|c|}
\hline \multirow[b]{2}{*}{ Strain } & \multicolumn{5}{|c|}{$\begin{array}{l}\text { Dehalogenase specific activity [units (mg protein })^{-1} \text { ] } \\
\text { (Dehalogenase activity ratio with respect to MCA) }\end{array}$} \\
\hline & MCA & DCA & $2 \mathrm{MCPA}$ & 22DCPA & 2MCBA \\
\hline $\begin{array}{l}\text { Continuous-flow culture } \\
\text { PP3 }\end{array}$ & $\begin{array}{c}0 \cdot 20 \\
(1.00)\end{array}$ & $\begin{array}{c}0 \cdot 19 \\
(0.93)\end{array}$ & $\begin{array}{c}0.08 \\
(0.44)\end{array}$ & $\begin{array}{c}0.04 \\
(0.21)\end{array}$ & $\begin{array}{c}0.03 \\
(0 \cdot 15)\end{array}$ \\
\hline $\begin{array}{l}\text { Culture at } \\
2500 \mathrm{~h}\end{array}$ & $\begin{array}{c}1 \cdot 29 \\
(1 \cdot 00)\end{array}$ & $\begin{array}{c}0 \cdot 29 \\
(0 \cdot 22)\end{array}$ & $\begin{array}{c}0.78 \\
(0.60)\end{array}$ & $\begin{array}{c}0 \cdot 43 \\
(0 \cdot 33)\end{array}$ & $\begin{array}{c}0 \cdot 31 \\
(0 \cdot 24)\end{array}$ \\
\hline $\begin{array}{l}\text { Closed culture } \\
\text { PP309 }\end{array}$ & $\begin{array}{c}0.31 \\
(1.00)\end{array}$ & $\begin{array}{c}0.10 \\
(0.32)\end{array}$ & $\begin{array}{c}0.15 \\
(0.48)\end{array}$ & $\begin{array}{c}0.09 \\
(0.29)\end{array}$ & $\begin{array}{c}0.07 \\
(0.23)\end{array}$ \\
\hline PP310 & $\begin{array}{c}0.27 \\
(1 \cdot 00)\end{array}$ & $\begin{array}{c}0.09 \\
(0.33)\end{array}$ & $\begin{array}{c}0.14 \\
(0.52)\end{array}$ & $\begin{array}{c}0.06 \\
(0.22)\end{array}$ & $\begin{array}{c}0.06 \\
(0.22)\end{array}$ \\
\hline $\begin{array}{l}\text { Inoculum } \\
\text { from continuous- } \\
\text { flow culture }\end{array}$ & $\begin{array}{c}0.37 \\
(1 \cdot 00)\end{array}$ & $\begin{array}{c}0 \cdot 12 \\
(0 \cdot 32)\end{array}$ & $\begin{array}{c}0.21 \\
(0.57)\end{array}$ & $\begin{array}{c}0.14 \\
(0.38)\end{array}$ & $\begin{array}{c}0 \cdot 10 \\
(0 \cdot 27)\end{array}$ \\
\hline
\end{tabular}

Table 2. Dehalogenase activity ratios in Pseudomonas putida from continuous-flow culture, as a function of dilution rate

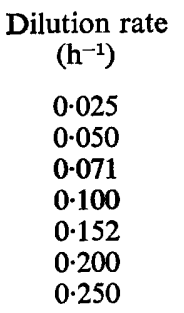

Dehalogenase activity ratio with respect to MCA

\begin{tabular}{ccccc}
\hline MCA & DCA & 2MCPA & 22DCPA & 2MCBA \\
1.00 & 0.22 & 0.47 & 0.34 & 0.26 \\
1.00 & 0.23 & 0.60 & 0.37 & 0.24 \\
1.00 & 0.23 & 0.50 & 0.30 & 0.25 \\
1.00 & 0.25 & 0.61 & 0.29 & 0.23 \\
1.00 & 0.26 & 0.67 & 0.37 & 0.25 \\
1.00 & 0.26 & 0.51 & 0.35 & 0.27 \\
1.00 & 0.27 & 0.42 & 0.31 & 0.25
\end{tabular}

culture (that is, a mixture of $P$. putida PP309 and PP310) were determined and compared with the original activities of $P$. putida PP3 (Table 1). The specific activities and major substrate activity ratios were markedly different. The specific activity of MCA dehalogenase showed a 6.5-fold increase, whilst activity against 2MCPA, 22DCPA and 2MCBA showed increases between 9.75 and 10.75 times the levels in $P$. putida PP3. In contrast, the DCA dehalogenase activity showed only a $1 \cdot 5$-fold increase. There were also major differences in the substrate activity ratios between $P$. putida PP3 and the $2500 \mathrm{~h}$ culture (Table 1). Initially, the MCA:DCA ratio in $P$. putida PP3 was nearly $1: 1$, whereas after the selection process this had changed to $1: 0 \cdot 2$. There was a slight increase in the ratio of 2MCPA, 22DCPA and 2MCBA with respect to MCA. However, there was no significant change in the ratio of 2MCPA:22DCPA:2MCBA which was $1: 0 \cdot 48: 0 \cdot 34$ for $P$. putida PP3 and 1:0.55:0.40 for the selected mixed culture. The dehalogenase activity ratios of both the selected strains grown separately in closed culture were similar to these values (Table 1).

The elevated levels of dehalogenase activity found in continuous-flow culture were not observed when strains PP309 or PP310 or the mixture taken directly from the chemostat were grown in closed culture on 2MCPA (Table 1). However, the dehalogenase specific activities against MCA, 2MCPA, 22DCPA and 2MCBA were significantly greater than those for the parent organism, strain PP3, grown under the same conditions. Moreover, the dehalogenase activity ratios observed in extracts of strains PP309 and PP310 grown in closed culture were similar to those of the $2500 \mathrm{~h}$ chemostat selected mixed organisms 


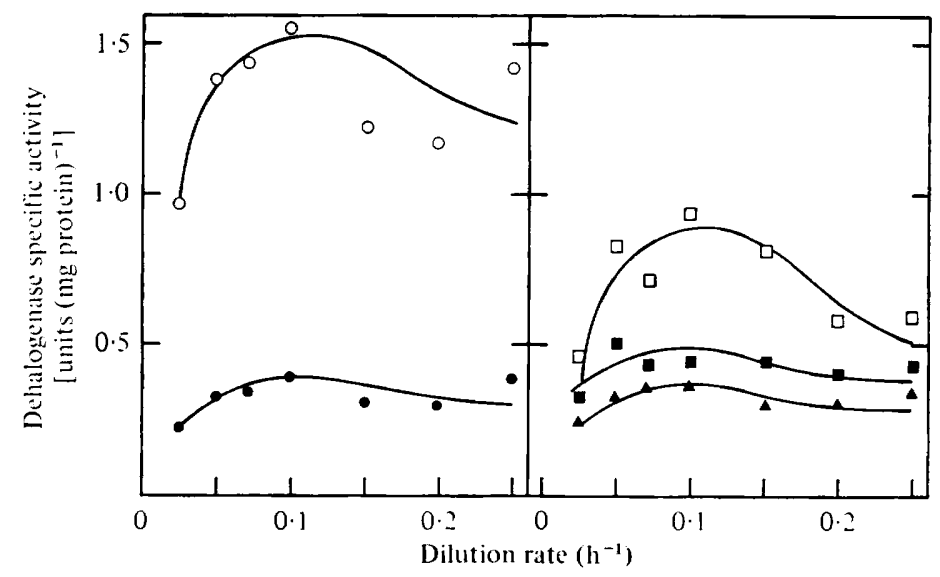

Fig. 2. Variation of dehalogenase specific activity with dilution rate in continuous-flow culture of Pseudomonas putida. Dehalogenase activity was measured against MCA (O), DCA (O), 2MCPA $(\square), 22 \mathrm{DCPA}(\square)$ and 2MCBA (A).

rather than the parent organism. Dehalogenase activity was absent in the mutants grown on succinate.

The pattern of dehalogenase specific activity as a function of dilution rate for the selected culture was examined (Fig. 2) and shown to be similar to the pattern previously observed for $P$. putida PP3 (Slater et al., 1979). A peak of activity against all five substrates examined was observed at $D=0 \cdot 10 \mathrm{~h}^{-1}$, a pattern characteristic for catabolic enzymes (Clarke \& Lilly, 1969; Dean, 1972). The dehalogenase activity ratios for MCA:DCA:2MCPA: 22DCPA:2MCBA remained constant, for all dilution rates examined, at 1:0.25 $\pm 0 \cdot 02$ : $0 \cdot 54 \pm 0 \cdot 09: 0 \cdot 33 \pm 0 \cdot 03: 0 \cdot 25 \pm 0 \cdot 01$, respectively (Table 2 ).

Slab gels run with extracts of organisms taken directly from the $2500 \mathrm{~h}$ selection experiment culture yielded two major bands with $R_{F}$ values of 0.41 and 0.78 when incubated with MCA, DCA or 2MCPA as the substrate. This was exactly the same pattern as was observed with extracts of the parent organism $P$. putida PP3. However, the slower moving band, that is, the fraction I dehalogenase, was much more densely stained than the more mobile band in extracts from the selection culture compared with extracts from the parent organism.

Both the selected strains, $P$. putida PP309 and PP310, were unable to grow in closed culture in defined media containing $2 \mathrm{MCBA}$ as the carbon source at various concentrations ranging up to $0.5 \mathrm{~g} \mathrm{Cl}^{-1}$. Moreover, both strains were unable to grow in defined media with 2MCPA as the carbon source if the initial concentration was greater than $0.3 \mathrm{~g} \mathrm{Cl}^{-1}$, although growth was extensive at lower concentrations. The parent organism, however, could grow well in defined medium containing 2MCPA at a concentration of $1.0 \mathrm{~g} \mathrm{C}^{-1}$. Throughout the selection experiment no strains of $P$. putida were isolated which could grow on plates or in closed culture with either 2MCBA or its putative breakdown product, 2-hydroxybutanoic acid, as the sole carbon source. Furthermore, when 2MCPA was removed from the fresh medium supply to the chemostat culture, washout occurred demonstrating that 2MCBA alone could not support growth.

\section{DISCUSSION}

The continuous-flow culture selection experiment failed to produce a strain of $P$. putida capable of growing on 2MCBA as the sole carbon and energy source. Although 2MCBA was significantly dechlorinated as a result of adequate dehalogenase activity, growth was not possible because the product, 2-hydroxybutanoic acid, failed to support growth. It 
appears from this experiment, however, that strains PP309 and PP310 were capable of utilizing some of the 2MCBA when grown in association with 2MCPA.

Despite the inability of the parent or mutant strains of $P$. putida to utilize 2MCBA, the selection experiments did result in the isolation of two mutants which had altered dehalogenase activities compared with the parent. This is an important conclusion since these experiments have demonstrated that mutants may be selected in the absence of the expected response, namely, the ability to use the previously non-growth substrate as a growth substrate. The observed results are consistent with the hypothesis that the increased specific activities and changed substrate activity ratios were due to an increased level of the fraction I dehalogenase with no change in the level of the fraction II dehalogenase. Previous work has shown that although both fraction I and fraction II dehalogenases possess activity against all the major substrates, the activity ratios vary considerably (Weightman et al., $1979 b$ ). Thus, changes in the level of one enzyme would be expected to have effects for all the substrates in proportion to their activity ratios. The increase in the MCA dehalogenase specific activity in the evolved chemostat culture, compared with the parent organism grown under the same conditions, was 1.09 units (mg protein) $)^{-1}$ (Table 1). If it is assumed that this increase in specific activity was due solely to an increase in the fraction I dehalogenase, then it would be expected that the DCA dehalogenase activity would show a $10 \%$ increase since the fraction I enzyme dehalogenates DCA at $10 \%$ of the MCA rate (Weightman et al., $1979 b$ ). Thus, the DCA dehalogenating capability of mutant strains would be expected to increase by $0 \cdot 11$ units (mg protein) $)^{-1}$ over the base level shown by the parent organism. On this basis an activity of 0.30 units (mg protein) ${ }^{-1}$ would be expected, a value which agrees closely with the observed activity of 0.29 units (mg protein $)^{-1}$. Thus, the $50 \%$ increase in DCA dehalogenase activity was entirely due to higher levels of fraction I dehalogenase, with no change in the level of fraction II dehalogenase.

Similarly, the intermediate increase in the MCA dehalogenase activity was due to the presence of two enzymes capable of dehalogenating MCA but with an increase in activity of only one of the enzymes. The MCA dehalogenase activity is almost equal in the two dehalogenases (Weightman et al., 1979 b). Thus, in the parent organism $50 \%$ of the observed MCA dehalogenase activity was due to the fraction I dehalogenase giving this enzyme a specific activity of $0 \cdot 1$ units (mg protein) $)^{-1}$. Again if it is assumed that all the increase in MCA dehalogenation capability was due to the fraction I dehalogenase, then the observed increase in activity, 1.09 units (mg protein) ${ }^{-1}$, represents a 10.9-fold increase in the activity of this enzyme. This figure is in close agreement with the increases found for 2MCPA, 22DCPA and 2MCBA, activities associated almost entirely with the fraction I dehalogenase.

The observed changes in activity ratios are also consistent with a greater expression of only the fraction I dehalogenase (Table 1) and these new activity ratios remained constant for different steady state cultures (Table 2). Furthermore, separation by gel electrophoresis confirmed that the mutant strains had a greater fraction I dehalogenase content compared with the fraction II dehalogenase.

The important question which remains unanswered at present is the exact nature of the mutation which causes the production of more fraction I dehalogenase in P. putida PP309 and PP310. The increased levels were not due to the selection of constitutive mutants (Hartley, 1974) since the two mutants failed to synthesize the dehalogenases in growth media lacking chlorinated alkanoic acid inducers. The mutation was, however, stable since the elevated fraction I dehalogenase activity relative to the fraction II activity was maintained in organisms grown and subcultured in closed culture. This contrasts with the unstable hyper-producing $\beta$-galactosidase mutants of Klebsiella aerogenes produced in lactoselimited chemostat culture which rapidly reverted to the parent phenotype levels in closed culture (Horiuchi et al., 1962). Furthermore, the mutation was not associated with a change in the enzyme's affinity for its substrates since the activity ratios for MCA, 2MCPA, 22DCPA and 2MCBA were constant. This contrasts with some of the pentitol dehydro- 
genase mutants of Klebsiella aerogenes selected in continuous culture (Hartley et al., 1972). In addition, the gel electrophoresis $R_{F}$ values for the two dehalogenases in the mutant strains were identical with the parent organism suggesting that the enzymes were unaltered.

It is possible that the higher fraction I dehalogenase activities were due to either improved rates of transcription and/or translation or to gene multiplication. The response of cultures to the external concentration of chlorinated alkanoic acids provides additional information which may indicate that gene multiplication could have occurred. We have previously shown that $P$. putida PP3 may be inhibited by chlorinated alkanoic acids (Slater et al., 1979) but that this strain was unaffected by $2 \mathrm{MCPA}$ at concentrations as high as $1.0 \mathrm{~g} \mathrm{Cl}^{-1}$ in the growth medium. It is likely that growth inhibition occurs as a result of intracellular substrate accumulation and that an important factor which determines the compound's growth inhibitory properties is the rate of substrate uptake. There are specific uptake mechanisms and we have shown, for example, that the inhibitory effect of brominated alkanoic acids on $P$. putida PP3 was greatly enhanced if the organisms were previously fully induced with 2MCPA (A. J. Weightman \& J. H. Slater, unpublished observations). Thus, the greater sensitivity of mutants PP309 and PP310 to relatively low 2MCPA concentrations $(0.3 \mathrm{~g} \mathrm{C}$ $\left.1^{-1}\right)$ suggests that, concomitant with the increased production of fraction I dehalogenase, there was an increase in the uptake system resulting in a more rapid rate of transport and accumulation of inhibitory levels of 2MCPA. This might have occurred if the gene multiplication affected both the catabolic enzyme and its uptake system.

A. J. W. thanks the Science Research Council and Shell Research for a CASE studentship. The work was also supported by a Royal Society grant to J.H.S.

\section{REFERENCES}

Bull, A. T., Senior, E. \& Slater, J. H. (1976). Concerning the evolution of metabolic capabilities in bacteria: Dalapon metabolism by Pseudomonas putida. Proceedings of Society for General Microbiology 4, 39.

Clarke, P. H. \& Lilly, M. D. (1969). The regulation of enzyme synthesis during growth. Symposia of the Society for General Microbiology 19, 113159.

DEAN, A. C. R. (1972). Influence of environment on the control of enzyme synthesis. Journal of Applied Chemistry and Biotechnology 22, 245-259.

Fairbanks, G., Steck, T. L. \& Wallach, D. F. H. (1971). Electrophoretic analysis of the major polypeptides of the human erythrocyte membrane. Biochemistry 10, 2606-2617.

Gornall, A. G., Bardawill, C. J. \& David, M. M. (1948). Determination of serum proteins by means of the biuret reaction. Journal of Biological Chemistry 177, 751-766.

Hartley, B. S. (1974). Enzyme families. Symposia of the Society for General Microbiology 24, 151182.

Hartley, B. S., Burleigh, B.D., Midwinter, G. G., MOORE, C. H., Morris, H. R., RigBY, P. W. J., Smith, M. J. \& TAYlor, S. S. (1972). Where do new enzymes come from? In Enzymes: Structure and Function, pp. 151-176. Edited by J. Drenth, R. A. Oosterbaan \& C. Veeger. Amsterdam: North-Holland.
Horiuchi, T., Tomizawa, J. \& Novick, A. (1962). Isolation and properties of bacteria capable of high rates of $\beta$-galactosidase synthesis. Biochimica et biophysica acta 55, 152-163.

KING, A. \& PHILlips, I. (1978). The identification of pseudomonads and related bacteria in a clinical laboratory. Journal of Medical Microbiology 11, 165-176.

LAEMMLI, U. K. (1970). Cleavage of structural proteins during the assembly of the head of bacteriophage T4. Nature, London 227, 680-685.

Senior, E., Bull, A. T. \& Slater, J. H. (1976). Enzyme evolution in a microbial community growing on the herbicide Dalapon. Nature, London 262, $476-479$.

Slater, J. H., Lovatt, D., Weightman, A. J., Senior, E. \& Bull, A. T. (1979). The growth of Pseudomonas putida on chlorinated aliphatic acids and its dehalogenase activity. Journal of General Microbiology 114, 125-136.

Weightman, A. J., Slater, J. H. \& Bull, A. T. $(1979 a)$. Cleavage of the carbon-chlorine bond by Pseudomonas putida. Society for General Microbiology Quarterly 6, 76-77.

Weightman, A. J., Slater, J. H. \& Bull, A. T. $(1979 b)$. The partial purification of two dehalogenases from Pseudomonas putida PP3. FEMS Microbiology Letters 6, 231-234. 